\title{
Compound Explosives Detection and Component Analysis via Terahertz Time-Domain Spectroscopy
}

\author{
Jindoo Choi, Sung Yoon Ryu, Won Sik Kwon, Kyung-Soo Kim, and Soohyun Kim* \\ Department of Mechanical Engineering, KAIST, Daejeon 305-701, Korea
}

(Received July 16, 2013 : revised September 2, 2013 : accepted September 17, 2013)

\begin{abstract}
We present qualitative and quantitative component analyses on compound explosives via Terahertz time-domain spectroscopy (THz-TDS) based on a combination of wavelet thresholding and wavelength selection. Despite its importance, the field of signal processing of $\mathrm{THz}$ signals of compound plastic explosives is relatively unexplored. In this paper, experiment results from explosives Composition B-3 and Pentolite are newly presented, suggesting a novel signal processing procedure for in situ compound explosives detection. The proposed signal processing method demonstrates effective component analysis even in noisy and humid environments, showing significant decrease in component concentration percentage error of approximately $22.7 \%$ for Composition B-3 and $48.8 \%$ for Pentolite.
\end{abstract}

Keywords : Terahertz time-domain spectroscopy, Wavelet, Compound explosives detection, Component
analysis

\section{INTRODUCTION}

Applications of Terahertz $(\mathrm{THz})$ radiation in spectral analysis [1], biology and medicine [2, 3], quality control [4], and communications [5] have spurred much interest in $\mathrm{THz}$ photonics. Furthermore, recent developments in $\mathrm{THz}$ technologies $[6,7]$ showed that $\mathrm{THz}$ waves could be a strong candidate for overcoming the obstacles associated with conventional explosives detection technologies, leading to extensive applications in security and military-related fields [7-10]. It should be noted that $\mathrm{THz}$ time-domain spectroscopy (THz-TDS) has been heavily utilized as the workhorse of the investigation of these capabilities of the $\mathrm{THz}$ regime, including the works on the detection and identification of both exposed and covered explosives through diffuse reflectance [11], investigation of the absorption spectra of numerous explosives and related compounds [12], and establishment of new spectral features within the frequency region of 3-6 THz $[13,14]$. Unfortunately, raw $\mathrm{THz}$ data obtained from THz-TDS cannot always be used for direct explosives detection applications. One of the major difficulties is highly related to the low signal-tonoise ratio (SNR) of the system setup. Additionally, $\mathrm{THz}$ absorption of water vapor and the reflections from the optical components of the system setup limit accurate spectral characteristic identification and analysis of the $\mathrm{THz}$ spectra [15-17].

Despite its importance, signal processing techniques of $\mathrm{THz}$ signals has drawn little attention in literature. Especially, signal processing of $\mathrm{THz}$ data based on wavelet transform is known to enhance signal quality without losing the essential information as compared to conventional filtering or smoothing algorithms [18]. Although research on utilizing wavelets in THz data post processing for gas sensing [19], and imaging $[20,21]$ have formerly been conducted, practical application on compound plastic explosives have rarely been reported, therefore they are still at an introductory level. Moreover, analyzing the 'recipe' of plastic explosives is known to help determine where the explosives were originally produced [22]. Therefore, there exists a huge area left for improvement in developing an overall signal processing procedure for in situ explosives detection and component analysis [23].

In this paper, we present experimental results regarding major interests and essential factors in compound explosives detection via THz-TDS: effective denoising, temporal and

Corresponding authors: *soohyun@kaist.ac.kr, ${ }^{\dagger}$ kyungsookim@kaist.ac.kr

Color versions of one or more of the figures in this paper are available online. 
spectral error handling, and practical component analysis schemes. THz spectra of Composition B-3 and Pentolite are investigated to verify the performance of the proposed procedures and algorithms on compound explosives.

\section{EXPERIMENT}

\subsection{THz-TDS System Setup}

Our experimental setup for THz-TDS is illustrated in Fig. 1. A Ti:Sapphire femtosecond laser is pumped by a continuous wave diode-pumped solid state laser at $532 \mathrm{~nm}$ wavelength. The femtosecond laser has a center wavelength of $790 \mathrm{~nm}$ (tunable by adjusting the slit position in the laser setup) and pulse duration of $25 \mathrm{fs}$.

The femtosecond pulse is divided into a pump and probe beam by a beam splitter. The optical pump beam $\left(250 \mathrm{~W} / \mathrm{cm}^{2}\right)$ stimulates $\mathrm{THz}$ pulsed radiation at the emitter, which is biased with a voltage of $20 \mathrm{~V}_{\mathrm{pp}}$. The $\mathrm{THz}$ emitter is a low-temperature-grown GaAs photoconductive antenna (Tera-SED10, Laser Quantum), with a dimension of 1 inch (O.D.) and no incorporated silicon lens. The diverging $\mathrm{THz}$ wave is collimated and focused onto the sample and recollected onto the receiver by using four off-axis parabolic mirrors. At the receiver $(1 \mathrm{~mm}$-thick ZnTe electro-optic crystal), the probe beam $\left(10 \mathrm{~W} / \mathrm{cm}^{2}\right)$ optically gates the detection with short pulse duration compared to the arriving $\mathrm{THz}$ pulse duration. Simple balanced detection is incorporated with a quarter-wave plate and a Wöllaston prism. A complete temporal scan of the $\mathrm{THz}$ signal is enabled by discrete micro-movement of

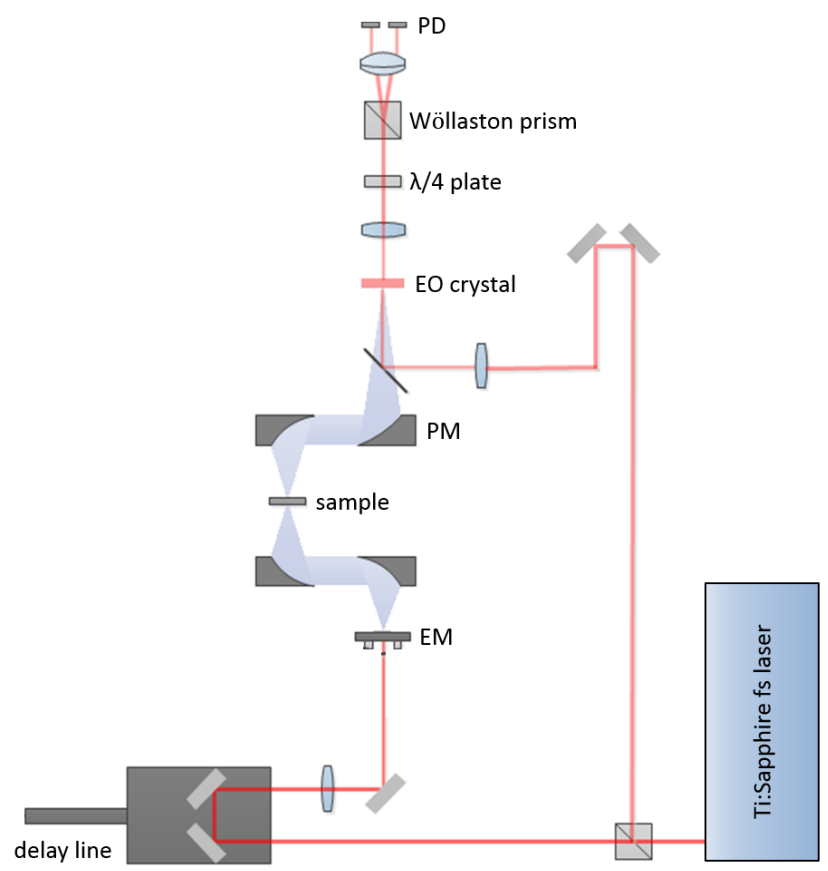

FIG. 1. Schematic diagram of the THz-TDS system setup. PD: photodetector, EM: THz emitter, PM: parabolic mirror. a motorized stage controlling the optical delay line. The THz-TDS system delivers a time-resolved $\mathrm{THz}$ pulse, where it is converted to a broadband $\mathrm{THz}$ spectrum via Fourier transform.

A discrete signal processing lock-in amplifier (LIA) was used for amplifying and denoising $\mathrm{THz}$ signals modulated by a function generator at $70 \mathrm{kHz}$. Different time constants were applied for the acquisition of different $\mathrm{THz}$ pulse SNR. Atmospheric attenuation was controlled by inserting dry nitrogen gas into an installable chamber which covered the THz generation and detection sections of the THz-TDS system.

\subsection{Sample Preparation}

Explosives samples (pentaerythritol tetranitrate; PETN, 1,3,5-trinitro-1,3,5-triazacyclohexane; RDX, and trinitrotoluene; TNT) were gently ground using a mortar and pestle to fine powder in accordance to the fact that compound explosives are made with highly explosive materials in powder form. The samples were compressed into pure pellets and compound pellets (all approximately $1 \mathrm{~mm}$ in thickness and $15 \mathrm{~mm}$ in diameter) directly using 10 tons of pressure with a hydraulic press. Two samples for compound explosives Composition B-3 and Pentolite - were produced by mixing the powder-form PETN, RDX, and TNT with the actual concentration ratio for making the above commercial plastic explosives. A mixture of $60 \%$ RDX and $40 \%$ TNT was used for Composition B-3, and 70\% PETN and 30\% TNT for Pentolite, following the composition information from [9]. The mass of each pellet was approximately $180 \mathrm{mg}$.

\section{DATA PROCESSING METHODS}

\subsection{Wavelet Thresholding}

The time-frequency localization characteristics of wavelet transform make it an effective representation of pulsed data [24]. Therefore, $\mathrm{THz}$ pulses, localized in both time and frequency, seem to be naturally suited to wavelet-based signal processing methods [19]. The defining equation of wavelet transform is presented as

$$
\psi_{j, n}(t)=\frac{1}{\sqrt{2^{-j}}} \psi\left(\frac{t-2^{-j} n}{2^{-j}}\right)=2^{j / 2} \psi\left(2^{j} t-n\right)
$$

where $\psi(t)$ denotes the wavelet, $j$ and $n$ are the scale and location indices, respectively. For most signal processing applications, the wavelet transform is best described in terms of filter banks, a group of filters that separate the input signal into multiple components. If the lowpass and highpass filter are chosen carefully, it is possible to reduce the length of the filtered signal by half and still be able to recover the original waveform [25].

Signal denoising is conducted by thresholding. In our work, we have chosen one of the most popular methods 
for denoising - hard-thresholding - defined by

$$
\rho_{T}(x)= \begin{cases}x & \text { if }|x| \geq T \\ 0 & \text { otherwise }\end{cases}
$$

where $T$ is the threshold value.

Additionally, since wavelet bases closely resemble $\mathrm{THz}$ waveforms, it is natural to assume that wavelets will outperform the conventional techniques. The applicability of this assumption to $\mathrm{THz}$ pulses of explosive materials is shown in the following sections of this paper.

\subsection{Wavelet Power Spectrum Estimation}

Although wavelet thresholding of the $\mathrm{THz}$ pulse shows competence in removing noise in the time-domain, it cannot effectively remove the fluctuations in the spectrum since the fluctuation does not come from noise, but rather from the long-lasting tail signal resulting from etalon reflections. The wavelet power spectrum estimation technique (WPSET) is a wavelet smoothing method of the log-scale periodogram of the obtained signal $[18,26]$, enabling effective spectral oscillation maintenance in the $\mathrm{THz}$ range.

The power spectrum of a THz signal $x[n]$ is described by

$$
S(f)=\sum_{n=-\infty}^{\infty} E\{x[m] x[m+n]\} e^{-i 2 \pi n f}
$$

where $E\{\cdot\}$ denotes the expectation. The periodogram of $x[n]$, which is often used as an estimate of the power spectrum $S(f)$ is defined as

$$
I_{N}(f)=\left|\sum_{n=0}^{2 N-1} x[n] e^{-i 2 \pi n f}\right|^{2}
$$

If $S(f)$ is sufficiently smooth and $N$ large, the periodogram and the power spectrum satisfy the following [26]:

$$
\ln I_{N}\left(\frac{k}{2 N}\right)-\gamma=\ln S\left(\frac{k}{2 N}\right)+\varepsilon(k), k=0,1, \cdots, N
$$

where $\gamma$ denotes the Euler-Mascheroni constant, approximately 0.5772 . Using equation (5), we can directly apply wavelet thresholding and remove the spectral fluctuations to an adequate level. The reconstructed power spectrum can be described by

$$
\ln S=\sum_{j=0}^{J} \sum_{n=0}^{N-1} \rho_{T}\left\{\left\langle\ln I_{N}, \psi_{j, n}\right\rangle\right\} \psi_{j, n}+\sum_{n=0}^{N-1} \rho_{T}\left\{\left\langle\ln I_{N}-\gamma, \phi_{J, n}\right\rangle\right\} \phi_{J, n}
$$

where $\psi[n]$ denotes the mother wavelet, and $\phi[n]$ the scaling function.

\subsection{Wavelength Selection}

Numerical data-analysis techniques have been developed which deal with selectively choosing the wavelengths for the desired use [27, 28]. Although these methods are capable of producing a highly accurate and complex analysis, they have not been widely utilized due to computational complexity and difficulty. We have investigated a means to select wavelengths in the $\mathrm{THz}$ regime for improved component analysis of compound explosives pursuing simplicity and practical ease.

After obtaining the $\mathrm{THz}$ spectra of the explosives samples, specific wavelengths are selected for each spectrum. Because the spectrum presents unique characteristics for each material, frequency ranges with high amplitude difference between other materials are chosen. This improves the orthogonality and independence from one another, making each component of the compound explosive into a pseudo-eigenvector. Additionally, since the chosen frequency ranges naturally exclude the absorption peaks from the water molecules, a digital compensation for the atmospheric degradation of $\mathrm{THz}$ data takes place. These wavelength-selected-spectra are then used for the spectroscopic evaluation of compound explosives.

\section{RESULTS AND DISCUSSION}

\subsection{Temporal Denoising of THz Pulses}

The performance of denoising via Daubechies wavelets (14 vanishing points, scale 2) [24] (by hard thresholding) was compared with that of Fourier denoising (lowpass filter with a cutoff frequency at $3 \mathrm{THz}$-spectral bandwidth of interest with a reliable SNR). A THz pulse signal of polyethylene (Sigma-Aldrich) was used for verification. A total SNR of $15 \mathrm{~dB}$ was computationally set with scaled additive white Gaussian noise, as shown in Fig. 2(a). Results show that noise reduction in the time-domain using wavelet thresholding (Fig. 2(b)) is superior to Fourier denoising (Fig. 2(c)). We can see from Table 1 that wavelet denoising showed approximately a $76 \%$ decrease in pulse correlation error which is significantly more effective than that of Fourier denoising, and a fourfold better pulse amplitude restoration. Correlation error is defined by

$$
e r r=1-\frac{E\left[(X-\bar{X})\left(X^{\prime}-\overline{X^{\prime}}\right)\right]}{\sigma_{X} \sigma_{X^{\prime}}}
$$

where $E$ denotes the expectation, $\sigma$ the standard deviation, $X$ and $X^{\prime}$ the original and denoised pulses, respectively.

Ultimately, THz pulses of PETN, RDX, and TNT were denoised via Daubechies wavelets. We could confirm that 

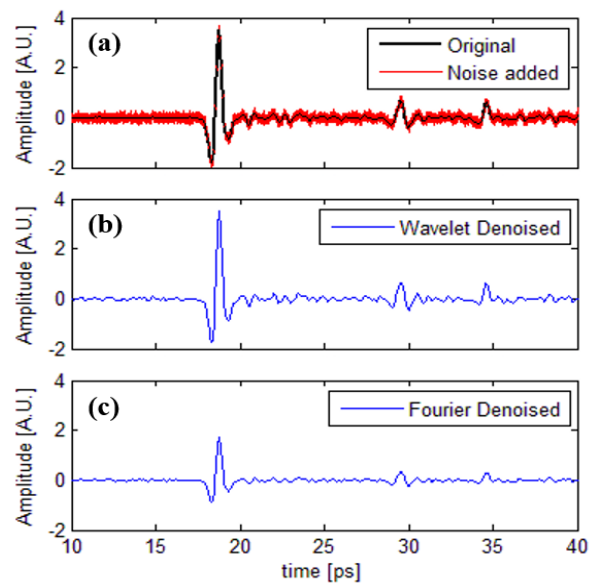

FIG. 2. (a) THz pulse of Polyethylene ( $\mathrm{SNR}=15 \mathrm{~dB}$ ), (b) Waveletdenoised pulse. (c) Fourier denoised pulse.

TABLE 1. Correlation error and RMS difference (between the original $\mathrm{THz}$ pulse) comparison of the wavelet denoised and Fourier denoised pulses

\begin{tabular}{c|c|c}
\hline \hline & Correlation error (\%) & RMS difference \\
\hline Noise added & 3.166 & 0.084 \\
\hline Wavelet denoised & 0.755 & 0.040 \\
\hline Fourier denoised & 1.036 & 0.165 \\
\hline
\end{tabular}
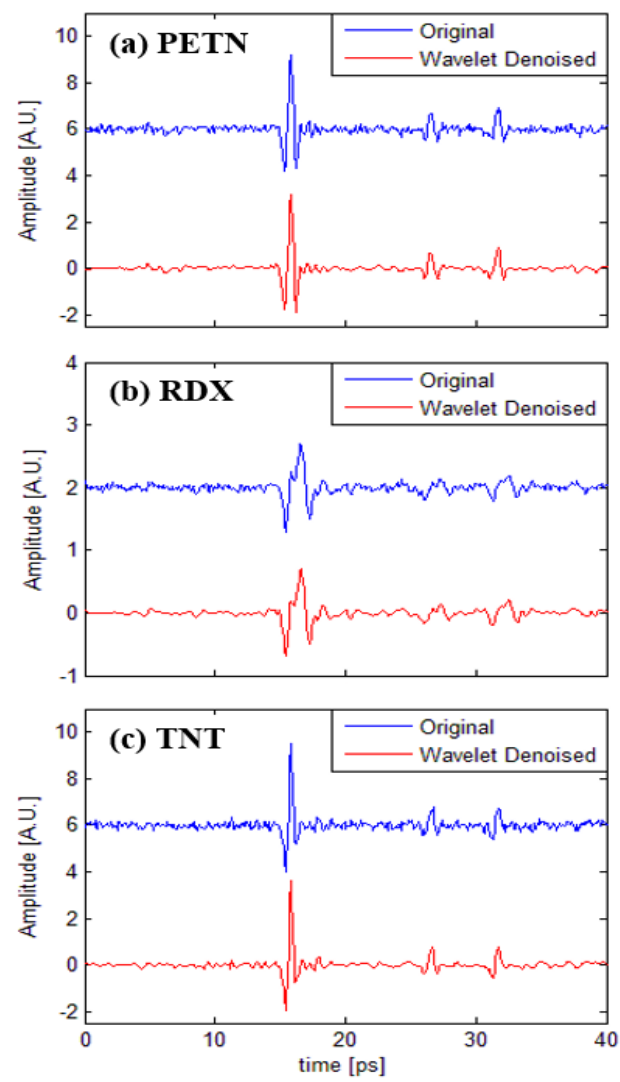

FIG. 3. Original (blue) and wavelet-denoised (red) THz pulses of (a) PETN, (b) RDX, and (c) TNT. wavelet-based techniques work effectively in terms of denoising and preserving pulse information of explosive samples, as presented in Fig. 3.

\subsection{Spectral Oscillation Maintenance}

In Fig. 3, we can see reflected echo pulses following the primary $\mathrm{THz}$ pulse, resulting from the etalon effect in samples and optical components (e.g. $\mathrm{THz}$ emitter and

TABLE 2. Characteristic peaks in the absorbance spectra of PETN, RDX, and TNT obtained by the WPSET, truncating method, and those reported in previous literature

\begin{tabular}{c|c|c|c|c|c|c|c|c}
\hline \hline \multicolumn{7}{c}{ Absorption peak position [THz] } \\
\hline \multicolumn{3}{c}{ PETN } & \multicolumn{3}{c}{ RDX } & \multicolumn{3}{c}{ TNT } \\
\hline $\begin{array}{c}\text { litera- } \\
\text { ture }\end{array}$ & $\begin{array}{c}\text { WPS } \\
\text { ET }\end{array}$ & $\begin{array}{c}\text { trunca- } \\
\text { tion }\end{array}$ & $\begin{array}{c}\text { litera- } \\
\text { ture }\end{array}$ & $\begin{array}{c}\text { WPS } \\
\text { ET }\end{array}$ & $\begin{array}{c}\text { trunca- } \\
\text { tion }\end{array}$ & $\begin{array}{c}\text { litera- } \\
\text { ture }\end{array}$ & $\begin{array}{c}\text { WPS } \\
\text { ET }\end{array}$ & $\begin{array}{c}\text { trunca- } \\
\text { tion }\end{array}$ \\
\hline 2.00 & 1.96 & 1.94 & 0.82 & 0.81 & 0.85 & 1.62 & 1.61 & - \\
\hline 2.16 & 2.20 & 2.22 & 1.05 & 1.03 & - & & 1.79 & 1.79 \\
\hline & & & 1.36 & 1.38 & 1.41 & 2.20 & 2.28 & 2.26 \\
\hline & & & 1.54 & 1.51 & 1.60 & & & \\
\hline & & & 1.95 & 1.98 & 1.98 & & & \\
\hline & & & 2.19 & 2.21 & 2.36 & & & \\
\hline
\end{tabular}
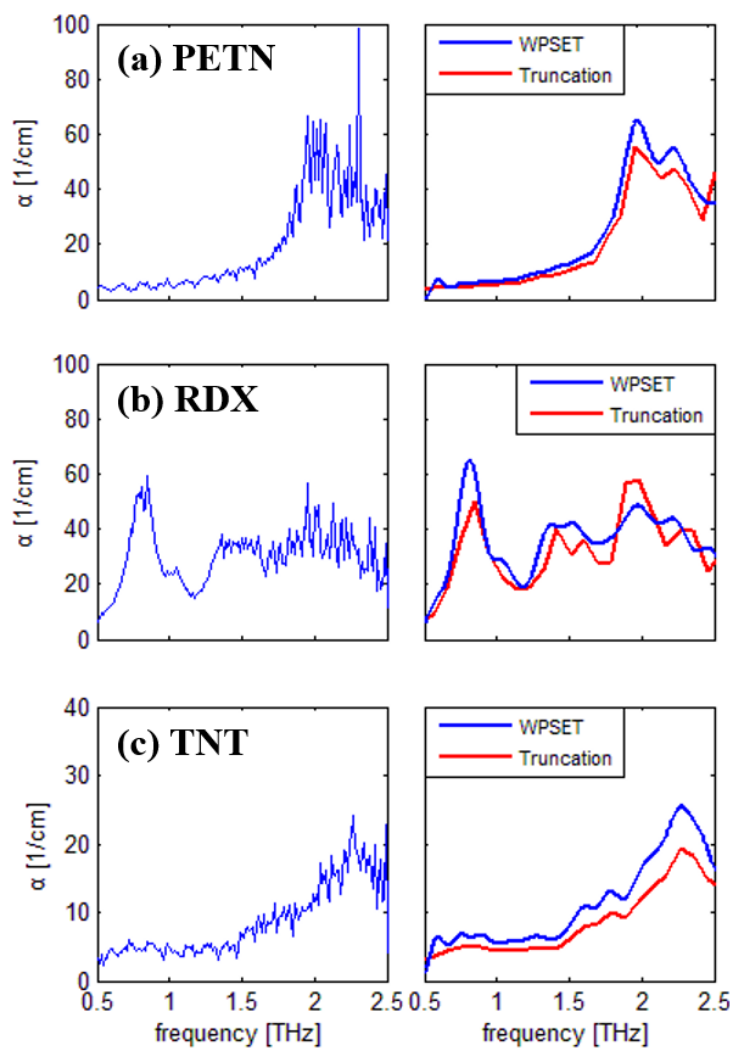

FIG. 4. THz absorbance spectra of (a) PETN, (b) RDX, and (c) TNT. The left row shows the originally obtained spectra and the right shows spectral oscillation-reduced spectra using the WPSET (blue) and truncating method (red). 
detector) in the THz-TDS setup. These secondary peaks give rise to spectral oscillations in the $\mathrm{THz}$ spectrum. In the algorithm level, the most conventional and widely used technique to remove such fluctuations is 'the truncating method', in which the time-domain data is truncated with the echoes excluded. Spectral oscillation maintenance was conducted using both the truncating method and the WPSET.

The characteristic peaks in the absorbance spectra of PETN, RDX, and TNT reported in previous literature [12] and those obtained by the WPSET and the truncating method are provided for direct comparison in Table 2 . From Table 2 and Fig. 4, we can see that the peak positions obtained from the two methods show difference. Although some peaks are well presented using the truncating method, the overall accuracy falls below from those of the WPSET. The second peak of RDX and the first peak of TNT could not be detected using the truncating method. On the other hand, it is clear that the WPSET not only reduced oscillations in the frequency-domain, but also facilitated accurate peak detection for the explosives samples. The obtained peak position data via the WPSET suggest its high credibility over the truncating method with regards to the accuracy, precision, and spectral resolution for component analyses of compound plastic explosives.

Ultimately, characteristic peaks in the $\mathrm{THz}$ absorbance spectra of Composition B-3 and Pentolite were identified through the WPSET, therefore providing a valuable prediction about the constituent materials. We could verify the presence of RDX and TNT from the absorbance peaks of Composition B-3 (Fig. 5(a)), and PETN and TNT from the peaks of Pentolite (Fig. 5(b)). Absorption peak positions shown in
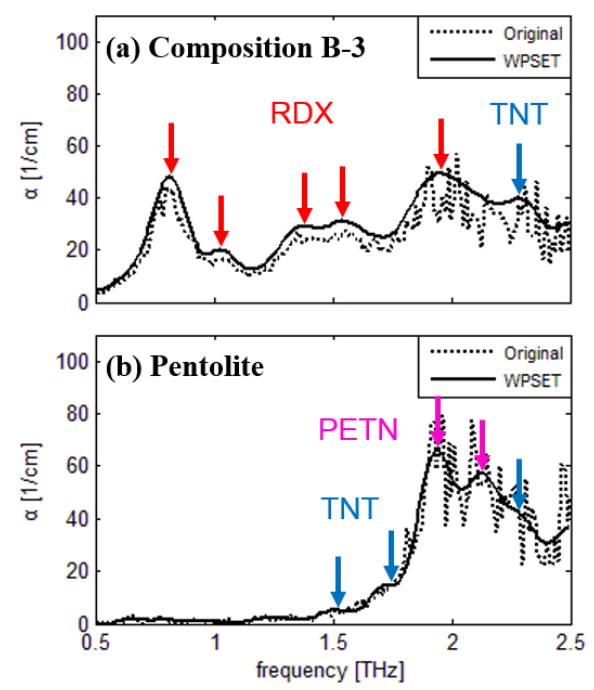

FIG. 5. THz absorbance spectra of (a) Composition B-3, and (b) Pentolite. The dotted line shows the originally obtained absorbance spectra and the solid line shows the spectra after using the WPSET. Arrows show absorption peaks originated from the constituent elements, where the red, blue, and magenta arrows point to the characteristic peaks of RDX, TNT, and PETN, respectively. the spectra of compound explosives were in good agreement with those of pure explosives.

\subsection{Component Analysis of Compound Explosives}

Component analyses of compound explosives Composition B-3 and Pentolite were first conducted with $\mathrm{THz}$ data gained in a laboratory-set fair environment. The compound explosive samples were purged in dry nitrogen for the acquisition of $\mathrm{THz}$ pulses without the high absorption of water vapor. Additionally, a relatively high SNR could be obtained by setting the time constant of the LIA to $1.3 \mathrm{~s}$. Fig. 6(a) and 7(a) respectively illustrate the concentrations of the constituent explosive materials in Composition B-3 and Pentolite.

Analyses were conducted not only in fair settings, but also in humid and noisy conditions. A low SNR (LIA time constant of $400 \mathrm{~ms}$ ) and relative humidity (RH) of $38 \%$ at $19.5^{\circ} \mathrm{C}$ were installed to visualize detection feasibility in real outdoor environments. Figures 6(b) and 7(b) show the amount of constituent elements in Composition B-3 and Pentolite in this setting. Furthermore, wavelength-selectedspectra of the compound explosives provided more accurate component analysis results, compensating for the humid
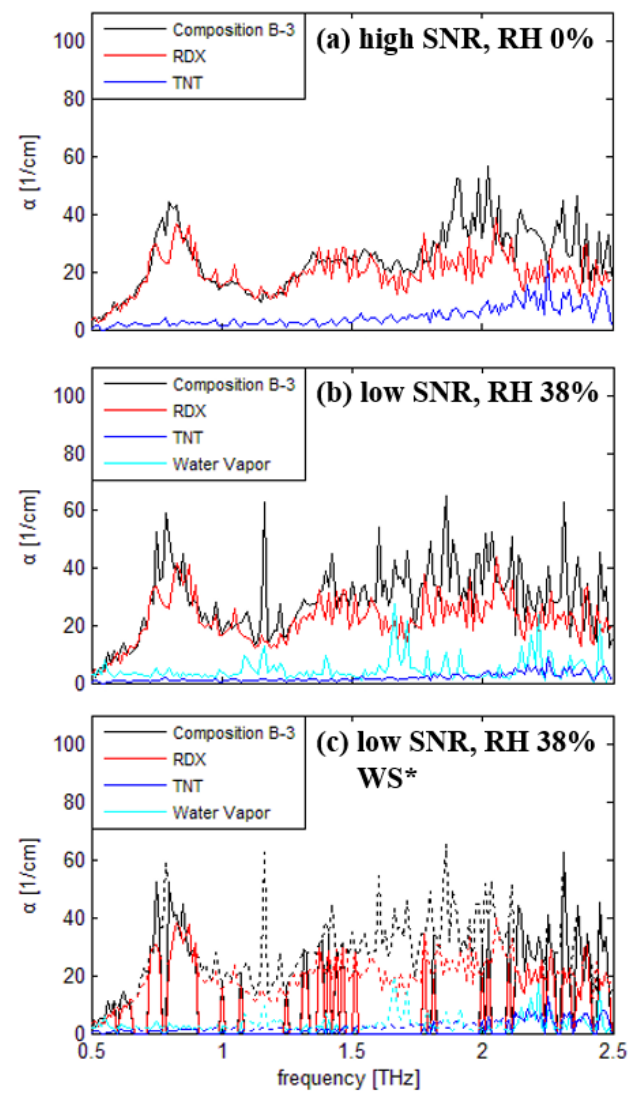

FIG. 6. RDX (red), TNT (blue), and water vapor (cyan) concentrations in Composition B-3 (black) in settings with (a) high SNR and RH 0\%, (b) low SNR and RH 38\%, and (c) low SNR and RH 38\% with wavelength selection (dotted: before wavelength selection, solid: after wavelength selection). * WS: wavelength selected. 

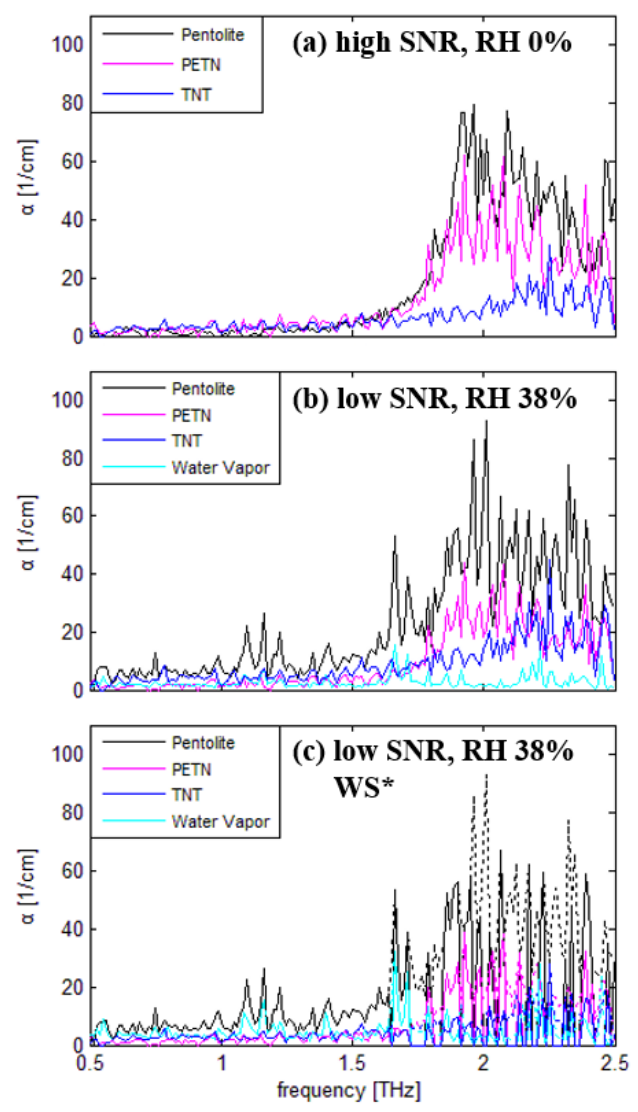

FIG. 7. PETN (magenta), TNT (blue), and water vapor (cyan) concentrations in Pentolite (black) in settings with (a) high SNR and RH $0 \%$, (b) low SNR and RH 38\%, and (c) low SNR and RH $38 \%$ with wavelength selection (dotted: before wavelength selection, solid: after wavelength selection). ${ }^{*}$ WS: wavelength selected.

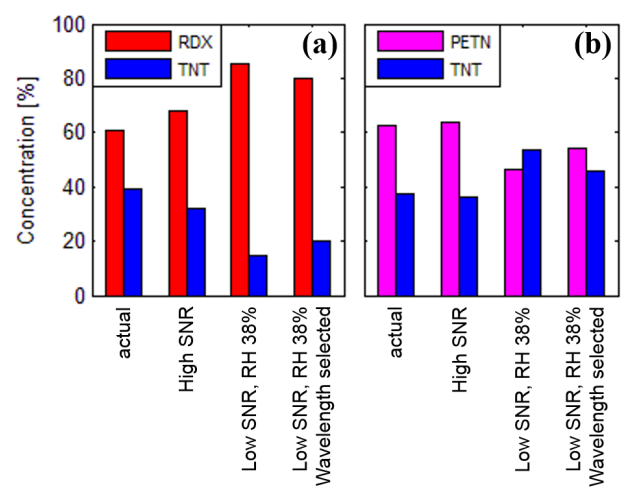

FIG. 8. Concentration percentages of the constituent elements of (a) Composition B-3 and (b) Pentolite for various experimental settings.

and noisy conditions (Figs. 6(c) and 7(c)).

The obtained concentration values of the constituent elements in the compound explosives are visualized in Fig. 8. We can see a huge concentration error in the humid and noisy environment, and the level of error decrease (approximately
TABLE 3. Concentration ratio error from the component analysis results of Composition B-3 and Pentolite

\begin{tabular}{c|c|c|c|c|c|c}
\hline \hline \multirow{2}{*}{} & \multicolumn{6}{|c}{ Concentration ratio error [\%] } \\
\cline { 2 - 7 } & \multicolumn{2}{|c}{ Composition B-3 } & \multicolumn{3}{c}{ Pentolite } \\
\hline $\begin{array}{c}\text { SNR } \\
\text { RH }\end{array}$ & $\begin{array}{c}\text { High } \\
/ 0 \%\end{array}$ & $\begin{array}{c}\text { Low } \\
138 \%\end{array}$ & $\begin{array}{c}\text { Low } \\
/ 38 \% \\
\text { WS* }\end{array}$ & $\begin{array}{c}\text { High } \\
/ 0 \%\end{array}$ & $\begin{array}{c}\text { Low } \\
/ 38 \%\end{array}$ & $\begin{array}{c}\text { Low } \\
/ 38 \% \\
\text { WS* }\end{array}$ \\
\hline PETN & - & - & - & 2.11 & 25.58 & 13.09 \\
\hline RDX & 11.90 & 41.08 & 31.75 & - & - & - \\
\hline TNT & 18.25 & 63.00 & 48.69 & 3.54 & 42.89 & 21.95 \\
\hline
\end{tabular}

* WS: Wavelength selected

22.7\% for Composition B-3 and 48.8\% for Pentolite) after wavelength selection. Table 3 shows the concentration error from the component analysis in detail.

\section{CONCLUSION}

We clearly presented experiment results regarding effective temporal denoising, spectral oscillation maintenance, and water vapor absorption compensation, suggesting an overall component analysis scheme for compound explosive materials. To the best of our knowledge, this is the first case where qualitative and quantitative component analyses on Composition B-3 and Pentolite were conducted. With enhanced SNR and effective peak detection via wavelet thresholding and the WPSET, straightforward spectral analyses could be conducted. Wavelength selection of each component spectrum improved the component analysis accuracy, compensating for the atmospheric degradation. This study shows the successful applicability of the proposed analysis scheme via THz-TDS to compound explosive materials. This is a dynamic field of research and further work must be done. Mathematical modeling of the $\mathrm{THz}$ pulse and optimized wavelength selection hold great potential for future research.

\section{ACKNOWLEDGMENT}

This work was supported by the National Research Foundation of Korea (NRF) grant funded by the Korean government (MEST) (No. 2012-0005646).

\section{REFERENCES}

1. C. Kang, C. Kee, I. Sohn, and J. Lee, "Spectral properties of THz-periodic metallic structures," J. Opt. Soc. Korea 12, 196-199 (2008).

2. P. H. Siegel, "Terahertz technology in biology and medicine," IEEE Trans. Microwave Theory Tech. 52, 2438-2447 (2004).

3. E. Jung, M. Lim, K. Moon, Y. Do, S. Lee, H. Han, H. 
Choi, K. Cho, and K. Kim, "Terahertz pulse imaging of micro-metastatic lymph nodes in early-stage cervical cancer patients," J. Opt. Soc. Korea 15, 155-160 (2011).

4. F. Rutz, M. Koch, S. Khare, M. Moneke, H. Richter, and U. Ewert, "Terahertz quality control of polymeric products," Int. J. Infrared Millimeter Waves 27, 547-556 (2006).

5. T. Kleine-Ostmann and T. Nagatsuma, "A review on terahertz communications research," J. Infrared Milli. Terahz. Waves 32, 143-171 (2011).

6. Y.-S. Lee, Principles of Terahertz Science and Technology (Springer, New York, USA, 2009), Chapter 3.

7. X.-C. Zhang and J. Xu, Introduction to THz Wave Photonics (Springer, New York, USA, 2010).

8. S. Caygill, F. Davis, and S. P. J. Higson, "Current trends in explosive detection techniques," Talanta 88, 14-29 (2012).

9. M. R. Leahy-Hoppa and M. J. Fitch, "Terahertz spectroscopy techniques for explosives detection," Anal. Bioanal. Chem. 395, 247-257 (2009).

10. H.-B. Liu, H. Zhong, N. Karpowicz, Y. Chen, and X.-C. Zhang, "Terahertz spectroscopy and imaging for defense and security applications," Proc. IEEE 95, 1514-1527 (2007).

11. H.-B. Liu, Y. Chen, G. Bastiaans, and X.-C. Zhang, "Detection and identification of explosive RDX by $\mathrm{THz}$ diffuse reflection spectroscopy," Opt. Express 14, 415-423 (2006).

12. J. Chen, Y. Chen, H. Zhao, G. Bastiaans, and X.-C. Zhang, "Absorption coefficients of selected explosives and related compounds in the range of 0.1-2.8 THz," Opt. Express 15, 12060-12067 (2007).

13. D. L. Woolard, E. R. Brown, M. Pepper, and M. Kemp, "Terahertz frequency sensing and imaging: A time of reckoning future applications?," Proc. IEEE 93, 1722-1743 (2005).

14. M. R. Leahy-Hoppa, M. J. Fitch, X. Zheng, L. M. Hayden, and R. Osiander, "Wideband terahertz spectroscopy of explosives," Chem. Phys. Lett. 434, 227-230 (2007).

15. M. van Exter, Ch. Fattinger, and D. Grischkowsky, "Terahertz time-domain spectroscopy of water vapor," Opt. Lett. 14, 1128-1130 (1989).

16. J. Kröll, J. Darmo, and K. Unterrainer, "High-performance terahertz electro-optic detector," Electron. Lett. 40, 763-764 (2004).
17. D. Turchinovich and J. I. Dijkhuis, "Performance of combined $<100>-<110>$ ZnTe crystals in an amplified THz timedomain spectrometer," Opt. Commun. 270, 96-99 (2007).

18. Y. Kim, K. H. Jin, J. C. Ye, J. Ahn, and D. Yee, "Wavelet power spectrum estimation for high-resolution terahertz time-domain spectroscopy," J. Opt. Soc. Korea 15, 103-108 (2011).

19. D. M. Mittleman, R. H. Jacobsen, R. Neelamani, R. G. Baraniuk, and M. C. Nuss, "Gas sensing using terahertz time-domain spectroscopy,” Appl. Phys. B 67, 379-390 (1998).

20. B. Ferguson and D. Abbott, "De-noising techniques for terahertz responses of biological samples," Microelectron. J. 32, 943-953 (2001).

21. B. Ferguson and D. Abbott, "Wavelet de-noising of optical terahertz pulse imaging data," Fluct. Noise Lett. 1, L65L69 (2001).

22. A. G. Davies, A. D. Burnett, W. Fan, E. H. Linfield, and J. E. Cunningham, "Terahertz spectroscopy of explosives and drugs," Mater. Today 11, 18-26 (2008).

23. J. Choi, S. Y. Ryu, W. Kwon, K.-S. Kim, and S. Kim, "Data processing of terahertz signals for in situ explosives detection and component analysis via terahertz time-domain spectroscopy," in Proc. The $37^{\text {th }}$ International Conference on Infrared, Millimeter and Terahertz Waves (IRMMW$\mathrm{THz}$ ) (University of Wollongong, Australia, Sep. 2013).

24. I. Daubechies, Ten Lectures on Wavelets (Society for Industrial and Applied Mathematics, Philadelphia, USA, 1992).

25. G. Strang and T. Nguyen, Wavelets and Filter Banks (Wellesley-Cambridge Press, Wellesley, MA, USA, 1997).

26. P. Moulin, "Wavelet thresholding techniques for power spectrum estimation," IEEE Trans. Sig. Proc. 42, 3126-3136 (1994).

27. P. J. Brown, "Wavelength selection in multicomponent nearinfrared calibration," J. Chemometrics 6, 151-161 (1992).

28. H. Sato, M. Kiguchi, F. Kawaguchi, and A. Maki, "Practicality of wavelength selection to improve signal-to-noise ratio in near-infrared spectroscopy," NeuroImage 21, 1554-1562 (2004). 